\title{
Adaptación y validación de la estructura factorial de las escalas de Felicidad Subjetiva Fluctuante y de Felicidad Subjetiva Duradera ${ }^{1,2}$
}

\author{
Adaptation and validation of the factorial structure \\ of the Scale of Subjective Fluctuating Happiness \\ and Subjective Authentic-Durable Happiness
}

\author{
José Luis Barrera-Gutiérrez, Denyzett Díaz-Ayala, \\ Juan José Sánchez-Sosa y Ana Moreno-Coutiño
}

Citación: Barrera G., J.L. Díaz A., D., Sánchez S., J.J. y Moreno C., A (2019). Adaptación y validación de la estructura factorial de las escalas de Felicidad Subjetiva Fluctuante y de Felicidad Subjetiva Duradera. Psicología y Salud, 29(2), 195-205.

RESUMEN

\begin{abstract}
Teóricamente, el modelo de felicidad egocéntrico/altruista propone que el logro de la felicidad está vinculado a la estructura del yo. Un funcionamiento egocéntrico induce fases de placer y displacer que se alternan repetidamente, dando como resultado una felicidad fluctuante. Por el contrario, un funcionamiento psicológico altruista postula un estado de felicidad duradera que depende menos de las circunstancias y que está relacionada con los recursos internos de una persona. Existen dos escalas para medir el constructo de felicidad a partir de este modelo teórico: la Escala de Felicidad Subjetiva Fluctuante y la Escala de Felicidad Subjetiva Duradera. No se cuenta en México con la adaptación y validación de estas escalas, por lo que el propósito de este trabajo fue traducir, adaptar culturalmente y evaluar la estructura factorial de las mismas mediante un análisis factorial confirmatorio. Como resultado, se obtuvo una escala final de 16 reactivos con dos factores: felicidad subjetiva duradera y felicidad subjetiva fluctuante. Se encontró que la estructura factorial está asociada al primero, por lo que es una herramienta confiable y válida.
\end{abstract}

Palabras clave: Felicidad; Felicidad fluctuante; Felicidad duradera; Pruebas psicométricas.

\begin{abstract}
Theoretically, the self-centered/selflessness happiness model proposes that the achievement of happiness is linked to the self structure. Self-centered functioning induces phases of pleasure and displeasure that alternate rapidly, leading to fluctuant happiness. In contrast, a selfless-centered psychological functioning induces a state of durable plenitude, which is less dependent on the circumstances and is related to the inner resources and abilities of a person. There are two scales to evaluate these kinds of happiness: the Fluctuant Subjective Happiness, and the Long-Lasting Happiness Scales. In Mexico, there is neither validation nor adaptation of these tools. Therefore, the aim of the present study was to translate, culturally adapt, and evaluate the factor structure of such scales through confirmatory factor analysis. A scale of 16 items was obtained with two factors: Long-lasting Happiness, and Fluctuant Subjective Happiness. The factor structure is associated with the first model, which suggests that this scale is a reliable and valid tool.
\end{abstract}

Key words: Happiness; Fluctuating happiness; Enduring happiness; Psychometric tests.

\footnotetext{
${ }^{1}$ Agradecimiento al Consejo Nacional de Ciencia y Tecnología (CONACyT) por el financiamiento para este estudio a través del CVU:323904, número de becario: 230035 con número de beca: 449025 .

${ }^{2}$ Los autores agradecen a los doctores Michaël Dambrun y Matthieu Ricard por su autorización para adaptar el cuestionario a población mexicana, así como a los doctores Ángel Eduardo Velasco Rojano y Carlos Alberto Mirón Juárez por su apoyo en el análisis estadístico de los datos. ${ }^{3}$ Facultad de Psicología, Universidad Nacional Autónoma de México, Av. Universidad 3004, Col. Copilco Universidad, Del. Coyoacán, Ciudad de México, México, correos electrónicos: psicopumatkd@hotmail.com, moca99_99@yahoo.com y denyzett_16@yahoo.com.mx. Artículo recibido el 4 de abril y aceptado el 9 de julio de 2018.
} 


\section{INTRODUCCIÓN}

$\mathrm{L}$ a investigación en la psicología ha estado más orientada a la identificación de trastornos mentales psicopatológicos, tales como ansiedad, depresión y neurosis, entre muchos otros, que a las bases del bienestar psicológico, la felicidad, la creatividad y otras fortalezas y virtudes humanas. No obstante, los estudios acerca de estos tópicos se han incrementado en las últimas décadas, dando nacimiento a la llamada psicología positiva. A pesar de los avances logrados acerca de la felicidad, los instrumentos para evaluarla son relativamente escasos. Entre los más utilizados se encuentran el Oxford Happiness Inventory (OHI), construido por Argyle, Martin y Crossland (1989); el Oxford Happiness Questionnaire, de Hills y Argyle (2002); la With Life Scale, desarrollada por Diener, Emmons, Larsen y Griffin (1985), y la Depresión-Happiness Scale, de Cammock y Lewis (1994). El OHI ha sido traducido al hebreo y aplicado en Israel por Francis y Katz (2000), y ha servido para construir el Chinese Happiness Inventory, administrado en Taiwán por Lu y Shih (1997).

En idioma español no hay suficientes instrumentos confiables y válidos para evaluar el constructo "felicidad". Entre los que más se citan se hallan los de Sánchez (1998), Anguas (2001), Albuquerque y Tróccoli (2004), Alarcón (2006) y Toribio, González, Valdez, González y Van Baneveld (2012). Como resultado de la falta de investigaciones sobre este tipo de instrumentos, puede haber confusiones en cuanto a la operacionalización del citado concepto, por lo que parece necesario que se continúen elaborando instrumentos sobre bases conceptuales sólidas. A esta motivación se suman las evidencias halladas sobre la influencia que ejercen los factores culturales, al punto que los instrumentos pierden sus propiedades psicométricas cuando se utilizan en culturas distintas a la del país de origen (Alarcón, 2006).

Tales hallazgos han planteado la necesidad de llevar a cabo una reevaluación rigurosa de las construcciones teóricas en que se basan los tests, por lo que en el presente estudio se recupera la propuesta de Dambrun y Ricard (2011), quienes se basan en el modelo teórico denominado Felicidad Egocéntrico/Altruista (Self-centeredness/Selfless- ness Happiness Model (SSHM), el cual sugiere que el logro de la felicidad está vinculado a la estructura del yo.

La percepción de un yo como una entidad permanente, independiente y sólida implica un funcionamiento psicológico egocéntrico que conduce a una felicidad fluctuante. Por otro lado, un funcionamiento psicológico altruista surge cuando la percepción del yo es flexible, lo que da como resultado una entidad que no es permanente, una estrecha conexión con el entorno y un comportamiento generoso, lo que favorece una felicidad auténtica y duradera.

De acuerdo al SSHM, en la percepción egocéntrica y del yo como una entidad permanente cada individuo tiene por objeto su conservación. La importancia exagerada dada al yo conduce a un principio "hedónico" (Higgins, 1997), mediante el cual los individuos están motivados para obtener placer y evitar el dolor, lo que crea una sensación de alegría y satisfacción; sin embargo, estos placeres son efímeros y dependen de las circunstancias externas; son, por tanto, inestables, y las sensaciones que evocan pronto se vuelven neutras (Wallace y Shapiro, 2006). Además, la imposibilidad de alcanzar ciertas expectativas da lugar a afectos aflictivos como la frustración, la ira, la hostilidad o los celos, todos los cuales dañan el bienestar (Miller, Smith, Turner, Guijarro y Hallet, 1996). En otras palabras, al tratar de maximizar el placer y evitar el dolor, el yo, como entidad permanente, induce una felicidad fluctuante en la que las fases de placer y displacer se alternan repetidamente.

Algunos teóricos proponen la existencia de una felicidad auténtica y duradera caracterizada por valores intrínsecos y prosociales (Ryan y Deci, 2001), tales como la gratitud (Peterson y Seligman, 2004), la sabiduría (Le, 2011) o el altruismo (Dambrun y Ricard, 2011). Este tipo de felicidad se entiende como una manera óptima de ser, como un estado de satisfacción duradera y de paz interior que, fundamentada en una cualidad de la conciencia que subyace e impregna cada experiencia, emoción y comportamiento, permite así aceptar tanto las alegrías como el dolor. El SSHM propone que la felicidad auténtica y perdurable se vincula íntimamente a un funcionamiento psicológico caracterizado por afectos benévolos, como la compasión y la empatía (Dambrun y Ricard, 2011). 
Para analizar la felicidad fluctuante y la duradera se necesita un instrumento de medición confiable y valido (Layard, 2010). A este respecto, las escalas SFHS y SA-DHS se han utilizado en entornos francófonos y anglófonos (Dambrun et al., 2012), hallándose en ambos casos evidencias de confiabilidad y validez adecuadas.

Ambas escalas cuentan con una buena confiabilidad probada por su consistencia interna: la SFHS obtuvo un coeficiente alfa de Cronbach de 0.89 , mientras que la SA-DHS uno de 0.93. De esta última escala se examinó asimismo la consistencia interna de las subescalas de Satisfacción (0.90) y Paz interior (0.87).

En cuanto a la validez de las escalas, los autores presentaron evidencias de validez convergente, reportando correlaciones significativas para la SA-DHS ( $r$ varió de 0.48 a 0.71 ) y moderadas para la SFHS ( $r$ varió de 0.24 a 0.48 ). Dicha validez se obtuvo correlacionando las escalas con un número de factores psicológicos asociados teórica y empíricamente con la felicidad, tales como optimismo (Scheier y Carver, 1985), autoeficacia (Bandura, 1991), sentido de coherencia (Antonovsky, 1987), resiliencia (Werner, 1992), mindfulness (Brown y Ryan, 2003), rumiación mental (Trapne11 y Campbell, 1999) y búsqueda del sentido de la vida (Steger, Frazier, Oishi y Kaler, 2006). Se encontró que las correlaciones eran moderadamente relevantes: de 0.22 a 0.53 para la SA-DHS y de 0.25 a 0.53 para la SFHS (Dambrun et al., 2012). Además, se obtuvo evidencia de validez convergente usando un marcador biológico (cortisol), el cual se ha asociado con el bienestar en otros estudios (Ryff et al., 2006). La única escala que correlacionó significativamente con esta hormona del estrés fue la SA-DHS $(r=-0.22, p<0.05)$.

México es considerado como uno de los países más felices del mundo, pues en diversos estudios ha puntuado por encima del promedio (Instituto Nacional de Estadística y Geografía, 2015; Figueroa, 2013; Fuentes y Reto, 2011; Olivares, 2015). Los datos revelan que la felicidad para los mexicanos tiene que ver con los valores tradicionales de la cultura, como la familia y el amor, mientras que factores como el ingreso económico o el puesto de trabajo son menos importantes.

Actualmente, se considera la felicidad como un indicador de progreso social y un objetivo de la política pública de numerosos gobiernos, México entre ellos. Por lo anterior, es necesario ampliar los conocimientos sobre la felicidad en general, y la de los mexicanos en particular. No obstante, se carece en el país de una herramienta que evalúe la felicidad según el modelo SSHM, por lo que el objetivo de este estudio fue determinar las propiedades psicométricas del instrumento de Dambrun y Ricard (2011) en una muestra heterogénea mexicana mediante un análisis factorial confirmatorio, para obtener los beneficios metodológicos, teóricos y prácticos que permitan ampliar el panorama acerca del constructo de felicidad, y contar así con una escala válida para otras investigaciones que la relacionen con diversas variables.

\section{MÉTODO}

\section{Participantes}

Se trabajó con cuatro grupos diferentes de participantes, uno en cada fase del estudio, para lo cual todos ellos dieron previamente su consentimiento.

El primer lugar, en el desarrollo de la traducción y adaptación culturalmente relevantes participaron seis jueces, todos ellos psicólogos con un grado mínimo de estudios de licenciatura y una amplia experiencia en el estudio de la felicidad, que además conocían el modelo teórico SSHM.

Un segundo grupo para el estudio piloto se conformó con veinte residentes de la Ciudad de México: diez mujeres y diez hombres, de entre 18 y 42 años de edad, con una media de 26.55 años y desviación estándar de 6.45, seleccionados de forma no probabilística intencional. El nivel educativo de los participantes fue como sigue: con primaria, uno $(5 \%)$, con secundaria, cinco $(25 \%)$, con bachillerato, siete $(35 \%)$ y con licenciatura, siete $(35 \%)$.

El tercer grupo, reunido para la validación psicométrica por medio del análisis factorial exploratorio (AFE), se conformó con 420 participantes mexicanos seleccionados por medio de un muestreo no probabilístico intencional, de los cuales 269 fueron mujeres (64\%) y 151 hombres (36\%) de entre 14 y 70 años de edad, con una edad media de 34.78 años y desviación estándar de 10.69, cuyo nivel educativo fue como se indica: primaria, 
$7(1.7 \%)$, secundaria, $51(12.1 \%)$, bachillerato, 61 (14.5\%), licenciatura, $180(42.9 \%)$, maestría, 104 (24.8\%) y doctorado, $16(3.8 \%)$.

El cuarto grupo se ocupó para el análisis factorial confirmatorio (AFC), hecho con 392 personas de nacionalidad mexicana seleccionadas de forma no probabilística intencional: 264 mujeres $(67.3 \%)$ y 128 hombres $(32.7 \%)$ de entre 14 y 70 años de edad, con una media de edad de 34.08 años y desviación estándar de 10.438. El nivel educativo de los participantes fue como sigue: primaria, $9(2.3 \%)$, secundaria, $48(12.2 \%)$, bachillerato, $43(11 \%)$, licenciatura, $181(46.2 \%)$, maestría, 87 (22.2\%), doctorado, $23(5.9 \%)$, y posdoctorado, $1(0.3 \%)$. Otras características sociodemográficas de los participantes se resumen en la Tabla 1.

Tabla 1. Características sociodemográficas de los participantes.

\begin{tabular}{|c|c|c|c|c|c|c|}
\hline \multirow[t]{2}{*}{ Característica } & \multicolumn{2}{|c|}{$\begin{array}{c}\text { Hombres } \\
n=279\end{array}$} & \multicolumn{2}{|c|}{$\begin{array}{c}\text { Mujeres } \\
\mathrm{n}=\mathbf{5 3 3}\end{array}$} & \multicolumn{2}{|c|}{$\begin{array}{c}\text { Total } \\
\mathrm{n}=\mathbf{8 1 2}\end{array}$} \\
\hline & M o frec. & D.E. $0 \%$ & M o frec. & D.E. $0 \%$ & M o frec. & D.E. O \% \\
\hline Edad & 35.11 & 11.11 & 34.10 & 10.26 & 34.44 & 10.57 \\
\hline \multicolumn{7}{|l|}{ Estado civil } \\
\hline Soltero & 116 & 41.6 & 260 & 48.8 & 376 & 46.3 \\
\hline Casado & 108 & 38.7 & 170 & 31.9 & 278 & 34.2 \\
\hline Unión libre & 40 & 14.3 & 65 & 12.2 & 105 & 12.9 \\
\hline Divorciado/separado & 14 & 5.0 & 33 & 6.2 & 47 & 5.8 \\
\hline Viudo & 1 & .4 & 5 & .9 & 6 & .7 \\
\hline \multicolumn{7}{|l|}{ Escolaridad } \\
\hline Primaria & 8 & 2.9 & 8 & 1.5 & 16 & 2.0 \\
\hline Secundaria & 69 & 24.7 & 30 & 5.6 & 99 & 12.2 \\
\hline Bachillerato & 48 & 17.2 & 56 & 10.5 & 104 & 12.8 \\
\hline Licenciatura & 92 & 33.0 & 269 & 50.5 & 361 & 44.5 \\
\hline Maestría & 49 & 17.6 & 142 & 26.6 & 191 & 23.5 \\
\hline Doctorado & 12 & 4.3 & 27 & 5.1 & 39 & 4.8 \\
\hline Posdoctorado & 1 & .4 & 1 & .2 & 2 & .2 \\
\hline \multicolumn{7}{|l|}{ Ocupación } \\
\hline Estudiante & 40 & 14.3 & 96 & 18.0 & 136 & 16.7 \\
\hline Obrero & 27 & 9.7 & 18 & 3.4 & 45 & 5.5 \\
\hline Oficio (carpintero, chofer, peluquero) & 22 & 7.9 & 11 & 2.1 & 33 & 4.1 \\
\hline Ama de casa & 0 & 0 & 40 & 7.5 & 40 & 4.9 \\
\hline Desempleado & 30 & 10.8 & 14 & 2.6 & 44 & 5.4 \\
\hline Comerciante & 28 & 10.0 & 10 & 1.9 & 38 & 4.7 \\
\hline Académico/investigador & 10 & 3.6 & 35 & 6.6 & 45 & 5.5 \\
\hline Psicólogo terapeuta & 70 & 25.1 & 183 & 34.3 & 253 & 31.2 \\
\hline Profesionista remunerado & 37 & 13.3 & 110 & 20.6 & 147 & 18.1 \\
\hline Pensionado & 4 & 1.4 & 4 & .8 & 8 & 1.0 \\
\hline Emprendedor & 11 & 3.9 & 12 & 2.3 & 23 & 2.8 \\
\hline
\end{tabular}

\section{Instrumentos}

Las versiones originales de las Escalas de Felicidad Subjetiva Fluctuante y Subjetiva Duradera fueron la Scale for Measuring Subjective Fluc- tuating Happiness (SFHS) y la Scale for Assessing Subjective Authentic-Durable Happiness (SA-DHS) (Dambrun et al., 2012). Tales instrumentos se desarrollaron en Francia a partir de información obtenida en un estudio piloto de una muestra de 320 
estudiantes universitarios, quedando conformados de la siguiente manera: la escala SFHS, con diez reactivos que se califican mediante una escala tipo Likert, de 1 ("fuertemente en desacuerdo") a 7 ("fuertemente de acuerdo"); en ella, un mayor puntaje refleja una mayor felicidad fluctuante. La escala SA-DHS contiene trece reactivos tipo Likert que se evalúan en un continuo de siete puntos que van de 1 ("muy bajo") a 7 ("muy alto"), así como dos subescalas: Satisfacción y Paz interior.

Para la traducción y adaptación culturalmente relevante se utilizó un formato para jueces con la versión original de las escalas en francés y una propuesta de traducción al español de México que incluía espacios para las propuestas de modificación. En la fase de piloteo y validación psicométrica se emplearon las versiones traducidas para población mexicana de las escalas de felicidad resultantes de la fase anterior. Así, la escala EFSD se conformó por trece reactivos con escala tipo Likert con siete opciones de respuesta que van de 1, "muy bajo", hasta 7, "muy alto". Por otra parte, la EFSF incluyó diez reactivos y una escala tipo Likert, con siete opciones de respuesta que van de 1, "completamente en desacuerdo", a 7, "completamente de acuerdo". De manera adicional, esta versión del cuestionario incluyó una sección breve para recolectar información demográfica de los participantes.

Para el AFC se empleó la versión validada en población mexicana producto de la fase anterior, con 22 reactivos organizados en dos dimensiones: Felicidad Subjetiva Duradera y Felicidad Subjetiva Fluctuante, así como una escala tipo Likert con siete opciones de respuesta, obteniéndose una varianza explicada de $60.07 \%$ y un índice de confiabilidad compuesta de 0.96 .

\section{Procedimiento}

Se llevó a cabo el procedimiento sobre la base de la propuesta metodológica-estadística de Reyes y García (2008) para la adaptación y validación psicométrica de las escalas SFHS y SA-DHS, consistente en traducción, validación por jueces, retraducción, adecuación, piloteo, revisión, aplicación a la población meta, validación psicométrica y análisis factorial confirmatorio.
La traducción fue efectuada por un traductor cuya lengua materna era el francés, hecho lo cual se entregaron a los jueces las versiones en español y francés, solicitándoles que indicaran si la consideraban adecuada o que sugirieran cambios. Al obtenerse entre los jueces $80 \%$ de acuerdo con la redacción final de cada reactivo, se realizó la retraducción al francés de la versión resultante, hecha por un segundo traductor que cumplía el perfil requerido de ser originario de Francia, no ser psicólogo de profesión y no conocer los instrumentos. Así, con base en los pasos anteriores se hicieron algunos cambios en la redacción para que se comprendieran mejor los reactivos en el piloteo, y asimismo se cambió el formato de respuesta de los instrumentos originales por una escala pictórica tipo Likert (cf. Reyes y García, 2008), con sus respectivas instrucciones para contestarlos. En ella, en lugar de ofrecer opciones de respuesta, se pusieron cuadros de diferente tamaño, considerándose que entre más grande fuese el cuadro, mayor sería el nivel de acuerdo con la descripción de la situación, y viceversa. El instrumento así conformado se aplicó a 20 participantes para verificar la comprensión de los reactivos, y una vez obtenidos los datos, se continuó el proceso de validación psicométrica del instrumento mediante el coeficiente de confiabilidad compuesta debido a que no es tan sensible al número de reactivos y tamaño muestral, a diferencia del coeficiente alfa. Para la fase del análisis factorial confirmatorio se solicitó a los participantes que respondieran nuevamente los instrumentos.

\section{RESULTADOS}

\section{Análisis factorial exploratorio}

Al realizarse el análisis de frecuencias de cada reactivo de la SFHS, se encontró que los diez reactivos la tuvieron en todas las opciones de respuesta. El análisis de curtosis y asimetría mostró una distribución sesgada en los reactivos. Se hizo la prueba U de Mann-Whitney para muestras independientes a fin de verificar la capacidad individual de discriminación de cada reactivo. El análisis de discriminación reveló que, de los diez reactivos, únicamente el número 1 ("He tenido satisfacciones 
y decepciones grandes") no resultó estadísticamente significativo, por lo que se excluyó de los análisis posteriores.

En la SA-DHS los trece reactivos mostraron frecuencias en todas y cada una de sus opciones de respuesta; su distribución, al igual que en la SFHS, resultó normal o sesgada. En virtud de la discriminación y direccionalidad de los reactivos no fue necesario eliminar ninguno (Tabla 2).

Tabla 2. Proceso de discriminación de los reactivos de la Escala de Felicidad Fluctuante integrada por dos subescalas.

\begin{tabular}{|c|c|c|c|c|}
\hline REACTIVO & Asimetría & U de Mann-Whitney & $\begin{array}{l}\text { Correlación } \\
\text { reactivo total }\end{array}$ & Criterio \\
\hline 1. Bienestar general & -0.54 & 0.00 & 0.70 & \\
\hline 2. Felicidad & -0.69 & 0.00 & 0.70 & \\
\hline 3. Placer & -0.72 & 0.00 & 0.70 & \\
\hline 4. Dicha completa & -0.68 & 0.00 & 0.78 & \\
\hline 5. Quietud (tranquilidad de espíritu) & -0.60 & 0.00 & 0.79 & \\
\hline 6. Satisfacción & -0.85 & 0.00 & 0.81 & \\
\hline 7. Serenidad & -0.58 & 0.00 & 0.73 & \\
\hline 8. Bendición & -1.10 & 0.00 & 0.61 & \\
\hline 9. Paz interior & -0.64 & 0.00 & 0.82 & \\
\hline 10. Realización & -0.64 & 0.00 & 0.72 & \\
\hline 11. Alegría & -0.92 & 0.00 & 0.74 & \\
\hline 12. Tranquilidad & -0.54 & 0.00 & 0.82 & \\
\hline 13. Plenitud & -0.67 & 0.00 & 0.89 & \\
\hline 1. He tenido satisfacciones y decepciones grandes. & -1.181 & .257 & 0.14 & Eliminado \\
\hline $\begin{array}{l}\text { 2. Mis periodos de placer son seguidos por periodos } \\
\text { de desagrado. }\end{array}$ & .490 & 0.00 & 0.67 & \\
\hline 3. Mi nivel de tranquilidad es cambiante. & .003 & 0.00 & 0.68 & \\
\hline $\begin{array}{l}\text { 4. Tengo periodos de euforia que son seguidos } \\
\text { por periodos de menos alegría. }\end{array}$ & .496 & 0.00 & 0.80 & \\
\hline 5. Puedo pasar de la euforia a la tristeza. & .807 & 0.00 & 0.78 & \\
\hline $\begin{array}{l}\text { 6. Mis periodos de bienestar van seguidos por periodos } \\
\text { de malestar. }\end{array}$ & .873 & 0.00 & 0.83 & \\
\hline $\begin{array}{l}\text { 7. Mi nivel de bienestar es inestable: a veces me siento } \\
\text { muy bien y a veces muy mal. }\end{array}$ & .660 & 0.00 & 0.84 & \\
\hline 8. Suelo ir de un nivel alto de placer a un nivel bajo. & 1.011 & 0.00 & 0.84 & \\
\hline $\begin{array}{l}\text { 9. Puedo sentirme totalmente pleno y después muy poco } \\
\text { satisfecho. }\end{array}$ & .772 & 0.00 & 0.82 & \\
\hline $\begin{array}{l}\text { 10. Puedo sentirme muy feliz y después muy triste, } \\
\text { todo el mismo día. }\end{array}$ & .832 & 0.00 & 0.79 & \\
\hline
\end{tabular}

Para verificar la adecuación de la matriz de correlaciones sobre la cual se basa el análisis factorial, se realizaron los análisis de medida de adecuación de Kaiser-Mayer-Olkin (KMO) y la prueba de esfericidad de Barttlet, obteniéndose $\mathrm{KMO}=.94 \mathrm{y}$ $X^{2}=5172.037, p=.000$, por lo que se concluyó que, en efecto, la había.

Debido a que el análisis de correlación entre los reactivos mostraba una predominancia moderada, se decidió hacer un análisis exploratorio de extracción de componentes principales con rotación oblicua a los 22 reactivos que no se eliminaron. A partir de este análisis, la escala quedó integrada por dos factores, con un porcentaje de varianza explicada de $60.07 \%$ y un índice de fiabilidad compuesta de .96 . Dichos factores fueron Felicidad Subjetiva Duradera (EFSD) y Felicidad Subjetiva Fluctuante (EFSF).

Estas subescalas pueden funcionar como escalas independientes. La EFSD, integrada por trece 
reactivos, explicó $33.64 \%$ de la varianza y obtuvo un índice de fiabilidad compuesta de .94 , mientras que la EFSF, integrada por nueve reactivos, expli- có $26.43 \%$ de la varianza, con un índice de confiabilidad compuesta de .93 (Tabla 3).

Tabla 3. Factorial exploratorio con rotación oblicua.

\begin{tabular}{|l|c|c|}
\hline \multicolumn{1}{|c|}{ Reactivo } & Factor $\mathbf{1}$ & Factor 2 \\
\hline 13. Plenitud & .838 & \\
\hline 6. Satisfacción & .793 & \\
\hline 4. Dicha completa & .790 & \\
\hline 12. Tranquilidad & .789 & \\
\hline 9. Paz interior & .776 & \\
\hline 5. Quietud & .753 & \\
\hline 11. Alegría & .743 & \\
\hline 2. Felicidad & .725 & \\
\hline 1.Bienestar general & .711 & \\
\hline 3.Placer & .708 & \\
\hline 10. Realización & .694 & \\
\hline 7. Serenidad & .686 & \\
\hline 8. Bendición & .591 & \\
\hline 8. Suelo ir de un nivel alto de placer a un nivel bajo. & & .843 \\
\hline 6. Mis periodos de bienestar van seguidos por periodos de malestar. & & .825 \\
\hline 7. Mi nivel de bienestar es inestable: a veces me siento muy bien y a veces muy mal. & & .807 \\
\hline 9. Puedo sentirme totalmente pleno y después muy poco satisfecho. & & .805 \\
\hline 4. Tengo periodos de euforia seguidos por periodos de menos alegría. & & .802 \\
\hline 5. Puedo pasar de la euforia a la tristeza. & & .794 \\
\hline 10. Puedo sentirme muy feliz y después muy triste, todo el mismo día. & & .783 \\
\hline 2. Mis periodos de placer son seguidos por periodos de desagrado. & & .649 \\
\hline 3. Mi nivel de tranquilidad es cambiante. & $\mathbf{9 3 4}$ & $\mathbf{6 9 3}$ \\
\hline \% de varianza explicada & $\mathbf{9 6}$ \\
\hline \% de varianza total explicada & $\mathbf{2 6 . 4 3 \%}$ \\
\hline Índice de fiabilidad compuesta & \\
\hline Índice de fiabilidad compuesta total de la escala de 22 elementos & \\
\hline
\end{tabular}

\section{Análisis factorial confirmatorio}

Debido a la falta de normalidad de los datos, se llevó a cabo un análisis factorial confirmatorio de máxima verosimilitud robusta (Schmitt, 2011) y se probaron dos dimensiones con 22 reactivos (Tabla 4).

Para la evaluación del ajuste del modelo se utilizaron los siguientes índices de bondad de ajuste: chi cuadrada normada $\left(\mathrm{X}^{2} \mathrm{~S}-\mathrm{B}[34]=2.506\right.$, $p=0.05$ ), dividida por los grados de libertad (siendo menor a 3.00 con una probabilidad no significativa), índice de ajuste comparativo de Bentler $(\mathrm{CFI}=0.963$ [para un ajuste apropiado los valores deben ser $\geq 0.95]$, y raíz cuadrada media del error de aproximación RMSEA (0.062) y SRMR (0.041 [para indicar un buen ajuste, los valores de estos índices deben ser $\leq 0.05]$ ).

La identificación del modelo se hizo fijando la varianza de los factores a 1; para esta estimación se utilizó el software $r$ con el paquete Latent Variable Analysis (LAVAAN, 0.5-22, 31 interacciones) (Rosseel, 2012), donde se contrastaron los índices de ajuste para el modelo. El modelo con dos dimensiones presentó índices aceptables, por lo que se considera que es la mejor versión de la escala. Los resultados se presentan en la Figura 1. 
Tabla 4. Valores del análisis factorial confirmatorio.

\begin{tabular}{|l|c|c|}
\hline \multicolumn{1}{|c|}{ Reactivo } & $\begin{array}{c}\text { Factor 1 } \\
\text { Felicidad } \\
\text { Duradera }\end{array}$ & $\begin{array}{c}\text { Factor 2 } \\
\text { Felicidad } \\
\text { Fluctuante }\end{array}$ \\
\hline 1. Bienestar general & $0.74(0.035)$ & \\
\hline 2. Felicidad & $0.82(0.030)$ & \\
\hline 3. Placer & $0.76(0.049)$ & \\
\hline 4. Dicha completa & $0.84(0.036)$ & \\
\hline 6. Satisfacción & $0.82(0.038)$ & \\
\hline 11. Alegría & $0.81(0.036)$ & \\
\hline 7. Serenidad & $0.68(0.074)$ & \\
\hline 13. Plenitud & $0.86(0.039)$ & \\
\hline 8. Suelo ir de un nivel alto de placer a un nivel bajo. & & $0.83(0.082)$ \\
\hline 3. Mi nivel de tranquilidad es cambiante. & & $0.60(0.175)$ \\
\hline 4. Tengo periodos de euforia seguidos por periodos de menos alegría. & & $0.74(0.122)$ \\
\hline 5. Puedo pasar de la euforia a la tristeza. & & $0.77(0.120)$ \\
\hline 6. Mis periodos de bienestar van seguidos por periodos de malestar. & & $0.77(0.086)$ \\
\hline 7. Mi nivel de bienestar es inestable: a veces me siento muy bien & & $0.81(0.111)$ \\
\hline y a veces muy mal. & & $0.81(0.104)$ \\
\hline 9. Puedo sentirme totalmente pleno y después muy poco satisfecho. & & $0.77(0.135)$ \\
\hline 10. Puedo sentirme muy feliz y después muy triste, todo el mismo día. & & $\mathbf{3 2 \%}$ \\
\hline \% de varianza explicada & $\mathbf{3 4 \%}$ & $\mathbf{9 1}$ \\
\hline Índice de fiabilidad compuesta & $\mathbf{9 3}$ & \\
\hline Índice de fiabilidad compuesta total de la escala de 16 elementos & $\mathbf{9 5}$ & \\
\hline
\end{tabular}

Figura 1. Análisis factorial confirmatorio de las escalas de Felicidad Subjetiva Duradera y Felicidad Fluctuante.

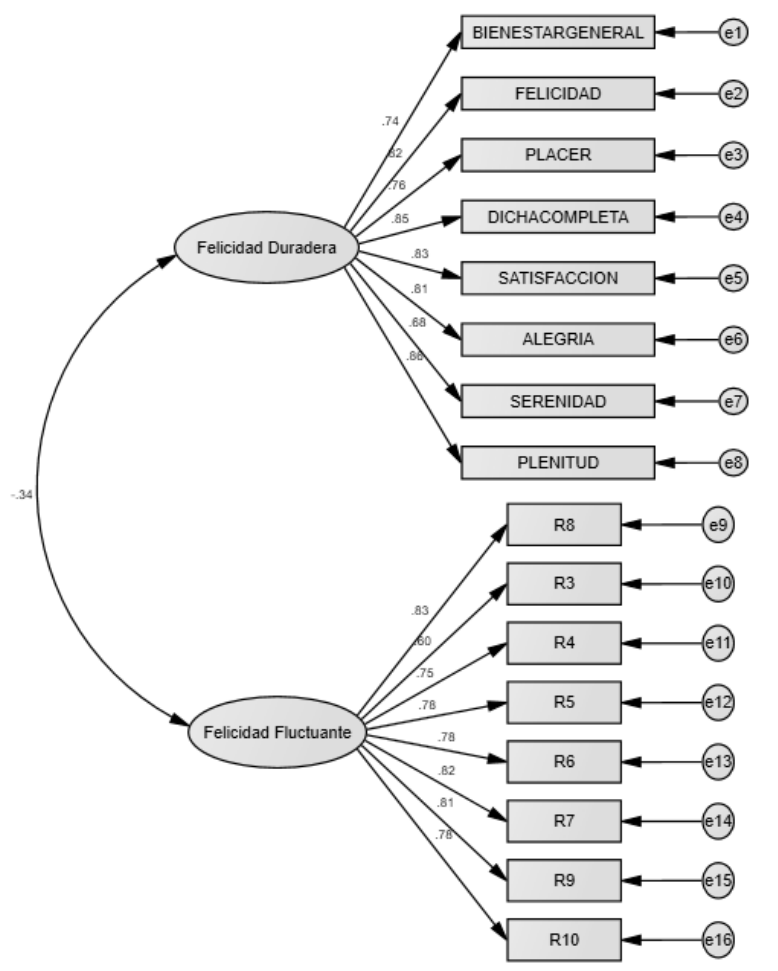

$\mathrm{X}_{\mathrm{S}-\mathrm{B}}^{2}(34)=2.506, p=0.05 . \mathrm{RMSEA}=0.062, \mathrm{SRMR}=0.041, \mathrm{CFI}=0.963$ 


\section{DISCUSIÓN}

El objetivo de este estudio fue evaluar la estructura factorial de las escalas de Felicidad Subjetiva Fluctuante y de Felicidad Subjetiva Duradera por medio de un análisis factorial confirmatorio. Los resultados señalan que el modelo de dos factores mostró un ajuste adecuado. La estructura factorial del instrumento así desarrollado está asociada con el modelo SSHM descrito por Dambrun y Ricard (2011).

El primer factor se denominó Felicidad Duradera. En este concepto, la felicidad está más estrechamente relacionada con valores intrínsecos y prosociales, conductas altruistas enfocadas a la autotrascendencia, en que se destacan los valores universales y la benevolencia. Está compuesta por los ítems 1 ("Bienestar general"), 2 ("Felicidad"), 3 ("Placer"), 4 ("Dicha completa"), 6 ("Satisfacción"), 11 ("Alegría"), 7 ("Serenidad") y 13 ("Plenitud").

El segundo factor, denominado Felicidad Fluctuante, se caracteriza por depender de las circunstancias externas, por lo cual está más estrechamente relacionado a una afectividad negativa, con una alternancia entre las fases de satisfacción e insatisfacción. Está compuesto por los ítems 8 ("Suelo ir de un nivel alto de placer a un nivel bajo"), 3 ("Mi nivel de tranquilidad es cambiante"), 4 ("Tengo periodos de euforia seguidos por periodos de menos alegría"), 5 ("Puedo pasar de la euforia a la tristeza"), 6 ("Mis periodos de bienestar van seguidos por periodos de malestar"), 7 ("Mi nivel de bienestar es inestable: a veces me siento muy bien y a veces muy mal"), 9 ("Puedo sentirme totalmente pleno y después muy poco satisfecho") y 10 ("Puedo sentirme muy feliz y después muy triste, todo el mismo día").

Los factores encontrados tienen un adecuado índice de confiabilidad compuesta de 0.93 para Felicidad Duradera y de 0.91 para Felicidad Fluctuante. El factor Felicidad Duradera es el que explica más varianza, lo que es congruente con la característica cultural mexicana de dar mayor importancia a valores internos y conductas prosociales -como contribuir a resolver las necesidades afectivas de la familia y los amigos-, que externos
(Díaz-Guerrero, 2003). El análisis factorial confirmatorio muestra que la estructura bidimensional obtenida se asemeja a la de las escalas originales de Dambrun et al. (2012). También se hallaron correlaciones estadísticamente significativas entre las dos dimensiones, con lo que se comprueba que cada reactivo corresponde a la dimensión correcta y mide lo que pretende medir. Así, a pesar de haber una moderada pero significativa correlación negativa entre las dos dimensiones, la felicidad auténtica duradera no parece ser simplemente el reverso de la felicidad fluctuante ya que, tal como se señaló antes, mientras que la primera está más estrechamente relacionada con la afectividad positiva y con la satisfacción con la vida, la segunda lo está más con la afectividad negativa.

Las dimensiones del instrumento constituyen una ventaja puesto que permiten identificar diferentes áreas de la felicidad de las personas que se encuentran afectadas por distintos factores internos o externos, lo que puede sugerir distintas medidas y tratamientos diferenciales para cada una de ellas. En particular, en los contextos clínicos puede ser una ayuda para identificar y dar tratamiento oportuno a los pacientes antes de que desarrollen diversos trastornos afectivos.

La extensión de la escala la convierte en una versión corta que incluye solamente a los reactivos con las mejores propiedades psicométricas para la población meta. Haber obtenido una versión así es una ventaja en razón de que es más práctica al aplicarse a personas con fatiga o con una comorbilidad asociada, ya que al paciente se le dificultaría contestar un instrumento extenso (Viner y Christie, 2005).

Por último, se recomienda realizar estudios con algún criterio externo que aporten otras evidencias de validez para las citadas escalas.

En conclusión, los resultados encontrados muestran que las escalas tienen propiedades psicométricas que las hacen confiables y válidas para evaluar población mexicana, constituyen un avance para el estudio de un constructo poco analizado en México, y brindan información relevante para diversas áreas de la psicología, tanto en la clínica como en la investigación. 


\section{REFERENCIAS}

Alarcón, R. (2006). Desarrollo de una escala factorial para medir felicidad. Revista Interamericana de Psicología, 40(1), 99-106.

Albuquerque A., S. y Tróccoli B., T. (2004). Desenvolvimiento de una escala de bienestar subjetivo. Psicología: Teoría e Pesquisa, 20, 153-164.

Anguas A., M. (2001). Identificación y validación del significado de bienestar subjetivo en México: Fundamentos para el desarrollo de un instrumento de medición. Revista Interamericana de Psicología, 35, 163-183.

Antonovsky, A.(1987). Untraveling the mastery of health: How people manage stress and stay well. San Francisco, CA: Jossey-Bass.

Argyle, M., Martin, M. y Crossland, J. (1989). Happiness as a function of personality and social encounters. En J. P. Forgas y J. M. Innes (Eds.): Recent advances in social psychological: an international perspective (pp. 189-203). Amsterdam: Elsevier.

Bandura, A.(1991). Self-efficacy mechanism in physiological activation and health promoting behavior. En J. I. Madden, S. Matthysee y J. Barchas (Eds.): Adaptation, learning, and affect (pp. 229-269). New York: Raven.

Brown, K. y Ryan, R.M. (2003). The benefits of being present: mindfulness and its role in psychological well-being. Journal of Personality and Social Psychology, 84(4), 822-848.

Cammock., T.J. y Lewis, Ch. (1994). Personality correlates of scores on the Depression-Happiness Scale. Psychological Reports, $75,1649-1650$.

Dambrun, M. y Ricard, M. (2011). Self-centeredness and selflessness: a theory of self-based psychological functioning and its consequences for happiness. Review General of Psychology, 15, 138-157.

Dambrun, M., Ricard, M., Després, G., Drelon, E., Gibelin, E., Gibelin, M. y Bray, E. (2012). Measuring happiness: from fluctuating happiness to authentic-durable happiness. Frontiers in Psychology, 3, 16. doi: 10.3389/fpsyg.2012.00016.

Díaz-Guerrero, R. (2003). Psicología del mexicano (6 $6^{\mathrm{a}}$ reimp.). México: Trillas.

Diener, E., Emmons, R.E., Larsen, R.J. y Griffin, S. (1985). The satisfaction with life scale. Journal of Personality Assessment, $49,71-75$.

Figueroa, H. (2013). “Querétaro, Monterrey y D.F, zonas más felices de México”. Diario Excelsior, 20 de febrero. Recuperado de http://www.excelsior.com.mx/nacional/2013/02/20/885054.

Francis, L.J. y Katz, Y.J. (2000). Internal consistency, reliability and validity of Hebrew translation of the Oxford Happinness Inventory. Psychological Reports, 87, 193-196.

Fuentes N., C. y Reto, F. (2011). Las condiciones de vida y la felicidad. En J. J. García y F. J. Sales (Eds.): Bienestar y calidad de vida en México (pp. 69-112). México: Centro de Estudios Sociales y de Opinión Pública.

Higgins, E.T. (1997). Beyond pleasure and pain. American Psychologist, 52(12), 1280-1300.

Hills, P. y Argyle, M. (2002). The Oxford Happiness Questionnaire: A compact scale for the measurement of psychological we11-being. Personality and Individual Differences, 33, 1073-1082.

Instituto Nacional de Estadística y Geografía (2015). Bienestar subjetivo. Contenido de la BIARE. México: INEGI. Recuperado de http://www.inegi.org.mx/inegi/contenid os/investigacion/experimentales/bienestar/ contenido.aspx.

Layard, R. (2010). Measuring subjective well-being. Science, 327, 534-535.

Le, T.N. (2011). Life satisfaction, openness value, self-transcendence, and wisdom. Journal of Happiness Studies, 12, 171-182.

Lu, L. y Shih, J.B. (1997). Personality and happiness: Is a mental health a mediator? Personality and Individual Differences, 31, 595-608.

Miller, T.Q., Smith, T.W., Turner, C.W., Guijarro, M.L. y Hallet, A.J.(1996). A meta-analytic review of research on hostility and physical health. Psychological Bulletin, 119, 322-348.

Olivares A., E. (2015). "Mexicanos tienen alto nivel de satisfacción y felicidad con la vida". La Jornada, 2 de junio. Recuperado de http://www.jornada.unam.mx/2015/02/06/sociedad/036n1soc.

Peterson, C. y Seligman, M.E.P. (2004). Character strengths and virtues: A Handbook and Classification. Washington, D.C.: American Psychological Association.

Reyes L., I. y García B., L.F. (2008). Procedimiento de validación psicométrica culturalmente relevante: un ejemplo. En S. Rivera A., R. Díaz-Loving, R. Sánchez A. e I. Reyes L. (Eds.): La Psicología Social en México, XII, 625-636. México: Asociación Mexicana de Psicología Social.

Rosseel, Y. (2012). Lavaan: An R package for structural equation modeling. Journal of Statistical Software, 48(2), 1-36.

Ryan, R.M. y Deci, E.L. (2001). To be happy or to be self-fulfilled: A review of research on hedonic and eudaemonic well-being. En S. Fiske (Ed.): Annual Review of Psychology (52; pp. 141-166). Palo Alto, CA: Annual Reviews, Inc.

Ryff, C.D., Love, G., Urry, H.L., Muller, D., Rosenkranz, M.A., Friedman, E.M. y Singer, B. (2006). Psychological well-being and ill-being: do they have distinct or mirrored biological correlates? Psychotherapy \& Psychosomatics, 75, 85-95.

Sánchez C., J. (1998). Escala de Bienestar Psicológico. Manual. Madrid: TEA Ediciones, S.A. 
Scheier, M.F. y Carver, C.S. (1985). Optimism, coping, and health: assessment and implications of generalized outcome expectancies. Health Psychology. 4, 219-247.

Schmitt, T.A. (2011). Current methodological considerations inexploratory and confirmatory factor analysis. Journal of Psychoeducational Assessment, 29(4), 304-321.

Steger, M.F., Frazier, P., Oishi, S. y Kaler, M. (2006). The Meaning in Life Questionnaire: assessing the presence of and search for meaning in life. Journal of Counseling Psychology. 53, 80-93.

Toribio, L., González N., I., Valdez, J.L., González, S. y Van Baneveld, H.O. (2012). Validación de la Escala de Felicidad de Alarcón para adolescentes mexicanos. Psicología Iberoamericana, 20(1), 71-79.

Trapnell, P.D. y Campbell, J.D. (1999). Private self-consciousness and the five-factor model of personality: distinguishing rumination from reflection. Journal of Personality and Social Psychology, 76, 284-304.

Viner, R. y Christie, D. (2005). Fatigue and somatic symptoms. British Medical Journal, 330(7498), $1012-1015$.

Wallace, B.A. y Shapiro, S.L. (2006). Mental balance and well-being: building bridges between Buddhism and Western psychology. American Psychologist, 61, 690-701.

Werner, E.E. (1992). The children of Kauai: resiliency and recovery in adolescence and adulthood. Journal of Adolescent Health, $13,262-268$. 